\title{
Transfer effects in part to whole free-recall learning
}

\author{
JAMES R. WHITMAN and E. PAT ANDERSON \\ Veterans Administration Hospital, American Lake, Tacoma, Wash. 98493
}

Two experimental groups learned a list of four visual figures; one group learned these to the criterion of two perfect trials, and the second group received additional practice. A control group received no learning trials with the figures. All groups $(\mathrm{N}=11 \mathrm{Ss}$ each) then received practice on a list of eight figures, which included those learned by. the experimental groups. Positive learning transfer was shown only by the group receiving the overtraining. Transfer was not accounted for by an increase in the relative frequency with which overlearned stimuli were recalled while learning the eight stimuli.

The purpose of this study was to determine whether or not free-recall learning is facilitated or made difficult when extensive prior practice is provided the learner on a substantial part of the stimuli included in the learning task. The responses produced during practice on the learning task are then analyzed in order to determine whether or not any observed learning transfer can be accounted for primarily on the basis of an increase in the probability with which the specific overlearned stimuli are recalled during the learning practice.

Garner (1962) has emphasized that free-recall learning involves the learning of the patterns of interrelationships (internal structure) that exist between the characteristics of a group of stimuli as these are perceived by the leamer, and secondly that this type of learning is not a question of the characteristics of individual stimuli, which in fact cannot be defined except by reference to the entire group in which the stimulus is embedded. Using nonsense words with which both the form and the amount of internal structure can be specified, Garner \& Whitman (1965) have shown that for both part-to-whole and whole-to-part free-recall learning, internal structure is a critical variable. In the above study the criterion used did not provide an opportunity for overlearning, and it is not clear how such additional training conducive to increased retention, modifies the process of free recall.

In an earlier study (Whitman, 1967), it was concluded that such additional practice increased the probability that overlearned stimuli would be recalled early in practice only. While a greater percentage of correct responses was produced with overlearning in that study, the difficulty of the learning task was such that few Ss were able to complete it. The present study is an extension of the above one by providing more extensive practice for the completion of the learning task and, hence, the expression of transfer effects. of the characteristics with equal frequency, and the internal structure of each part was in the form of simple contingencies between variables. The experimental groups learned one of these parts (Part A) prior to practice on the larger set of eight figures (Part A plus Part B).

\section{GENERAL PROCEDURE}

Prior to practice with the visual figures, each $S$ was given a digit symbol task with a $90-\mathrm{sec}$ practice period. He was then told that he was going to learn to reproduce from memory several figures that would be shown to him one at a time. He was told what the four characteristics of the figures were and that with these characteristics 16 different figures could be produced. A sample set of the 16 possible figures was then shown $S$ for $3 \mathrm{sec}$, after which he was required to draw the entire set from instructions read to him by $E$, introducing one variable at a time. The set he had constructed was checked for completeness and then removed by $\mathrm{E} . \mathrm{S}$ then received the instructions appropriate for the group to which he had been assigned prior to the start of the experiment.

$S s$ in one control group were required to reconstruct from memory, with assistance from $E$ when necessary, the complete set of stimuli for a second time. After their productions were collected, they were then asked to draw any four of the figures they had just completed. Instructions and learning trials with the eight stimuli (long list) then began.

The Ss in two experimental groups were told that they were going to learn to reproduce from memory four of the figures from the set just constructed and that the figures would be shown one at a time. They were told that the order in which the figures were shown was not important and would change for each presentation and also that they could recall the figures in figures in each represented all values of all

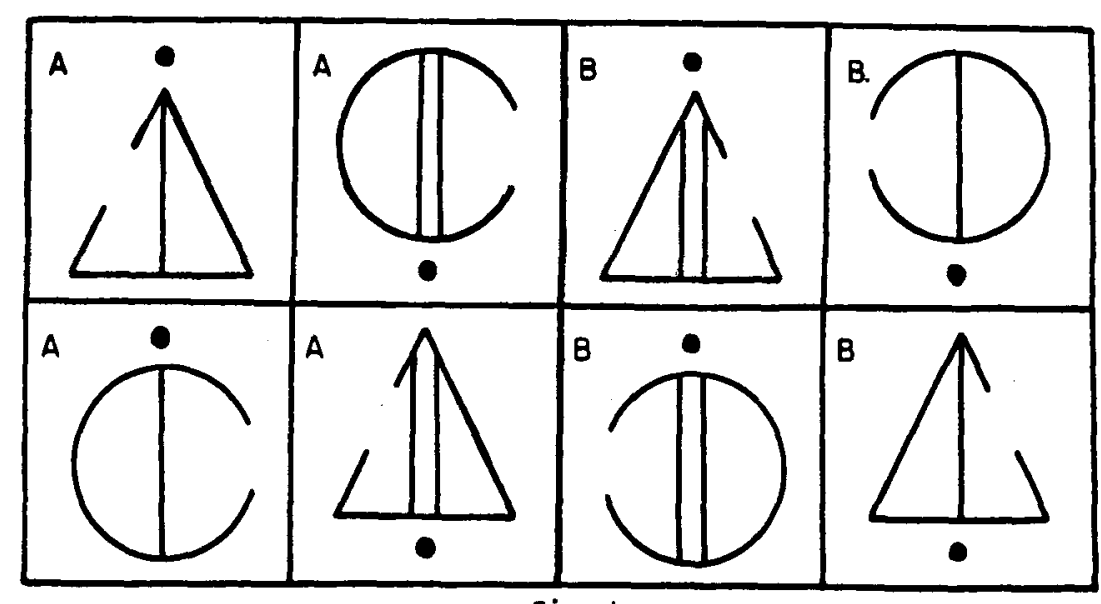

Fig. 1

Fig. 1. The stimuli used in the experiment. They are labeled here only, to indicate those in short list (A) and the additional ones (B), which were added to form the long list. 


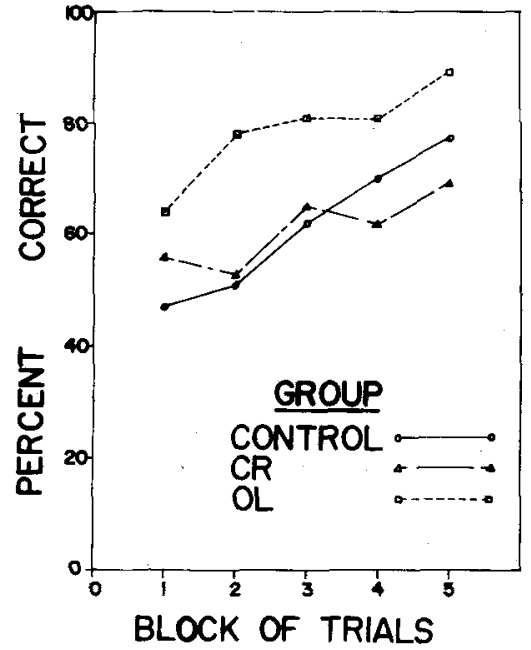

Fig. 2. Learning curves based on number of correct responses, averaged for blocks of three trials each.

any order. Further, they were told that they would not know how many of their answers were correct until they had correctly recalled all four figures on two successive trials. Learning trials with the four figures (short list) were then administered. All trials consisted of one presentation of the stimuli followed by an answer period.

After reaching criterion with the short list, one of the two experimental groups (CR group) received the instructions and learning trials with the long list, and the second (OL group) received 15 additional trials with the short list. Instructions for this additional practice were, after five additional free-recall trials, to reproduce each time not only the figures, but also the order in which they were shown, which, as before, changed for each of the last 10 trials. Instructions for practice on the long list began for this group after these trials.

Instructions for practice on the long list informed $S$ that: (1) he was now to learn eight different figures which would be presented one at a time; (2) he would be shown the list twice each time before he would be asked to recall them; (3) the order was not important and would not be important on any later trial; and (4) he would not know how many of his answers were correct until he reached the criterion of two perfect trials. $S$ was told the rate at which the stimuli were to be presented and the time allowed for the answer period. Ss in both experimental groups were also told that they would not know prior to practice whether or not the figures they had just learned were included in the list they were about to learn. A trial with the long list consisted of two presentations of the list of stimuli, with a 10 -sec pause between list presentations, followed by an answer period.

Practice for all Ss continued on the long list until the criterion of two perfect trials was reached or for 15 trials, whichever came first. After this practice, $S$ s in the experimental groups were required to recall the four stimuli in the short list, and Ss in the control group were required to reproduce any four figures from the long list.

For each trial with both the short and the long lists a different random order for presentation of stimuli was used. Except for the additional practice received by the OL group, each stimulus was presented for $5 \mathrm{sec}$, one immediately following another; all answer periods for the short list were $30 \mathrm{sec}$ and for the long list, $60 \mathrm{sec}$. For the overlearning trials a stimulus was presented for $3 \mathrm{sec}$, and the answer period lasted $20 \mathrm{sec}$.

\section{RESULTS}

The Ss were assigned randomly to the groups. However, a measure of the comparability in learning ability of the two experimental groups was provided by the performance of these groups in learning the short list. The mean number of trials to reach the criterion of two perfect trials with the short list was 8.3 and 7.3 for the $\mathrm{CR}$ and $\mathrm{OL}$ groups, respectively. The difference was not significant $(t<1$, $\mathrm{df}=20$ ).

The effect of the prior training with the short list was inferred from three measures of performance on the long list and also from an analysis of the relative frequency with which stimuli from the short list were recalled during such practice. Three scores

Table 1

Measures of Performance After Practice on Longer List, by Group

\begin{tabular}{|c|c|c|c|}
\hline \multirow[b]{2}{*}{ Measure } & \multicolumn{3}{|c|}{ Group } \\
\hline & $\begin{array}{l}\text { Control } \\
(\mathrm{N}=11)\end{array}$ & $\begin{array}{c}\mathrm{CR} \\
(\mathrm{N}=11)\end{array}$ & $\begin{array}{c}\mathrm{OL} \\
(\mathrm{N}=11)\end{array}$ \\
\hline Ss reaching criterion $(\%)$ & 45 & 18 & 73 \\
\hline Trials to criterion (Mdn) & $15+$ & $15+$ & 6.9 \\
\hline Mean percent correct & 62 & 61 & 79 \\
\hline Mean number of errors & 40 & 45 & 21 \\
\hline \multirow{2}{*}{$\begin{array}{l}\text { Mean percentage of A stimuli } \\
\text { in correct responses* }\end{array}$} & & & \\
\hline & 50 & 52 & 57 \\
\hline
\end{tabular}

*Based on performance up to but not including criterion trials. from learning trials with the long list were obtained for each S: (1) number of trials to reach the criterion of two perfect trials, (2) mean number of correct responses per trial, and (3) mean number of errors per trial. The Mann-Whitney $U$ test was used with the first scores, since some Ss failed to reach criterion. The analysis of variance was used with each of the other two scores. For all tests of significance, two-tailed tests and the $\mathrm{p}<.05$ level were used.

Scores for the groups are shown in Table 1, which also includes the percentages of Ss reaching criterion in each group. Figure 2 shows learning curves for the three groups based on the mean number of correct responses per trial, with perfect recall assumed for $S$ after criterion was reached.

$\mathrm{Ss}$ in the OL group gave more correct responses and made fewer errors during practice than did those in the control or CR groups, which did not differ significantly on these two measures. Also, Ss in the OL group reached criterion in fewer trials than did those in the other two groups, which were combined for this analysis since few Ss in each of the two groups had reached criterion with the practice provided. The conclusion is that overlearning the short list facilitated the learning of the long list and that learning the short list to the criterion of two perfect trials did not.

The relative frequency with which stimuli from the short list were recalled while learning the long list was measured by determining for each $S$ the percentage of correct responses that were stimuli in the short list. These percentages were determined for all responses produced prior to reaching criterion and for those Ss not reaching criterion, all responses during the practice provided. The mean percentage for each group is shown in Table 1. These scores were not normally distributed, and $70 \%$ of the values were between 45 and 55. The Mann-Whitney $U$ test was used for group comparisons, and none of the group differences was significant ( $p>.20$ for all comparisons). This evidence accordingly indicates that the learning transfer shown by the $O L$ group is not accounted for by an increase in the probability with which the overlearned stimuli from the short list were recalled during practice.

After practice on the long list, Ss in the experimental groups were asked to recall the stimuli from the short list, and those in the control group recalled any four stimuli. No $S$ in the $C R$ group recalled all of the $A$ stimuli. The mean number of A stimuli recalled by this group (1.6), when compared with that for the control group, 
was no better than chance $(\mathrm{t}=1.07$, $\mathrm{df}=20$ ). Five of $10 \mathrm{Ss}^{1}$ in the OL group recalled all four stimuli in the short list. These Ss, who showed perfect retention for the stimuli in the short list, were among those in their group who reached criterion on the long list. The relative frequencies with which stimuli from the short list were recalled while learning the long list were $50 \%$ for four of these Ss and $65 \%$ for the fifth. Their recall while learning the long list were therefore not solely a matter of the availability in memory of the stimuli from the short list.

\section{DISCUSSION}

There are two conclusions from the present study. The first is that free-recall learning is facilitated when overlearning is provided $S$ on a substantial part or subset of the learning task and, secondly, that such learning transfer is not accounted for primarily on the basis of an increase in the probability with which the previously learned subset of stimuli are recalled during practice. Since the difficulty of the learning task used in this study has been shown to be related to the form and amount of internal structure produced by the interrelationships existing in this group of stimuli (Whitman \& Garner, 1963), it appears likely that the transfer effect produced with overlearning reflects a change in the variables characterizing the stimuli as these are perceived by S. Such a change would also change the internal structure in the group of stimuli constituting the long list, and since the transfer is positive, the changed internal structure would be one that facilitates free-recall learning. The change suggested here refers to how $S$ perceives the stimuli in the learning task and would be analogous to the change that occurs when, in language learning, combinations of letters become meaningful as words.

Tulving (1966) interprets the transfer in free recall as involving the reorganization of memory units into higher order units. The material in the learning task he used consisted of meaningful words. He found negative transfer in several part-to-whole learning studies, and the learning curves shown in his report as reflecting this negative transfer are similar to those for the control and CR groups in the present study (see Fig. 2). He has suggested that subjective units were formed during the part learning which was inappropriate in the subsequent learning. Bower \& Lesgold (1969) replicated Tulving's result with single-word presentation also using meaningful words, but they also found that part-to-whole transfer was highly positive when such list words were organized or grouped for $S$, with the whole list organization being compatible with the part list organization.

For these investigators the unit of analysis is the individual stimulus, and not the entire group of stimuli in the learning task, and the memory units are groups of stimuli. But the present study with visual figures shows that with overlearning and the consequent greater ability to recall a substantial part or group of the stimuli in the learning task, the recall of the group of overlearned stimuli is not significantly greater than it would be without such prior training, and still positive learning transfer occurs. This result is not consistent with an interpretation of learning transfer in which the unit for analysis is the individual stimulus.

The type of transfer in this study, however, may differ from that produced in the studies of Tulving (1966) and Bower \& Lesgold (1969), whose Ss learned meaningful words. The difference between these two classes of material in the complexity of the variables descriptive of each, as well as the findings of Bower \&
Lesgold (1969) would suggest that such a distinction is required.

\section{REFERENCES}

BOWER, G. H., \& LESGOLD, A. M. Organization as a determinant of part to whole transfer in free recall. Journal of Verbal Learning \& Verbal Behavior, 1969, 8, 501-506.

GARNER, W. R. Uncertainty and structure as psychological concepts. New York: Wiley, 1962.

GARNER, W. R., \& WHITMAN, J. R. Form and amount of internal structure as factors in free recall learning of nonsense words. Journal of Verbal Learning \& Verbal Behavior, 1965, 4 , 257-266.

TULVING, E. Subjective organization and effects of repetition in multi-trial free-recall learning. Journal of Verbal Learning \& Verbal Behavior, 1966, 5, 193-197.

WHITMAN, J. R., \& GARNER, W. R. Concept learning as a function of form of internal structure. Journal of Verbal Learning \& Verbal Behavior, 1963, 2, 195-202.

WHITMAN, J. R. In W. Wathen-Dunn (Ed.), Models for the perception of speech and visual form. Cambridge: M.I.T. Press, 1967. Pp. 279-281.

\section{NOTE}

1. Recall from one $S$ was excluded due to E's error.

\title{
Acoustic vigilance behavior
}

\section{in four-year-old children*}

\author{
JOHN L. LOCKE \\ Children's Research Center, Champaign, Ill. 61820
}

Eighty 4-year-old Ss were given an acoustic vigilance task in which they were to signal the presence of pure tones which appeared irregularly, infrequently, and at faint suprathreshold levels. In several significant aspects these young Ss behaved similarly to adults. Acoustic vigilance testing is discussed as a potentially useful framework in which to assess and observe children's attentional behavior.

Vigilance is essentially that behavior observable when Ss attempt to identify the presence of signals which are faint, infrequent, and irregular. It is a potentially useful measure of human behavior because it seems to require the kind of global attention which apparently underlies performance on most tasks, and it appears to be relatively uncontaminated by the requirement of higher-level perceptual or cognitive processing.

Vigilance research, promoted mainly by military and industrial interests, seems mostly to have dealt with adults' attention to visual signals (e.g., watch keeping, quality-control inspection). Children's information processing, on the other hand, depends heavily upon the input of acoustic stimuli, and it would be helpful if some measure of their acoustic vigilance behavior were available. This paper reports an initial attempt to develop a task permitting the controlled observation of acoustic vigilance. Specifically, we were interested

*This paper was supported by Public Health Research Grant MH-07346 from the National Institute of Mental Health. The author acknowledges the assistance of Jeffrey Goldstein in gathering and analyzing data. 